\title{
Management of Pain in Opioid Addicted Patients: An Education Plan for Psychiatric Nurses
}

\author{
Janice M. Parker ${ }^{1}$ \\ ${ }^{1}$ Ascension Borgess Hospital, Kalamazoo, Michigan, USA \\ Correspondence: Samuel P. Abraham, Associate Professor of Nursing, 1001 Bethel Circle, Bethel University \\ School of Nursing, Mishawaka, Indiana, 46545, USA.
}

Received: April 9, 2019

doi:10.20849/ijsn.v4i2.572
Accepted: April 30, 2019

Online Published: May 5, 2019

URL: https://doi.org/10.20849/ijsn.v4i2.572

\begin{abstract}
Background: Identified on an acute care behavioral health unit has been a gap in nursing education and knowledge about opioid addiction and pain management. Nurses are often frustrated that there seems to be no clear way to manage acute pain in individuals suffering with opioid addiction. Because of difficult behavior sometimes displayed in those with opioid addiction, nurses may be prone to attitudes that reflect bias on their part when administering care. The psychiatric nurses need to learn about the disease model of addiction so its premise can be shared with the opioid addicted patients and their families. Purpose: The purpose of this proposal is to create a plan to teach the Behavioral Health nurses about opioid addiction and management of acute pain to help those afflicted with opioid addiction. Plan: The plan for addressing the gap includes education about opioid addiction, the barriers presented by stigma and bias, and the use of opioid replacement therapy to manage acute pain in those with opioid addiction. Patient education will focus on addiction as a disease and the impact of stigma on those with the disease. Implementation: Implementation of the project will include the development of two classes about management of acute pain in opioid addicted patients emphasizing education of the nurse and patient as the prime solution to this dilemma. The classes will stress the need to model conversations with psychiatric physicians to develop strategies to manage acute pain in patients with opioid addiction. Conclusion: Opioid replacement therapy seems to be the only answer for individuals in need of rehabilitation to end their burden of addiction. It should be accessible to any person with addiction to opioids in every healthcare setting.
\end{abstract}

Keywords: management of pain, opioid addiction, psychiatric, education plan, psychiatric nurses

\section{Introduction}

The concept of presenting a class about pain management for opioid addicted patients was born out of requests to the Behavioral Health (BH) Nurse Educator from hospital medical nurses. These nurses wanted to receive education about "managing the drug seekers." This gave rise to speculation the BH unit nurses would appreciate education around acute pain management of opioid addicted patients as well. Indeed, the inpatient psychiatric nurses voiced several concerns about their opioid addicted patients. Similar to the feelings of the hospital medical nurses, the psychiatric nurses' challenge was to provide a positive patient experience. This was thwarted by behaviors exhibited by patients with opioid addiction. The difficulties encountered were of concern to nurses on several levels. Presentation of a class about pain management in opioid addicted patients to the psychiatric nurses is anticipated as a precursor to eventual hospital-wide education classes on this topic to more than 700 nurses. The purpose of this proposal is to create a plan to teach the Behavioral Health nurses about opioid addiction and management of acute pain to help those afflicted with opioid addiction.

In describing the background, the Behavioral Health (BH) unit is a 50 bed, short-term psychiatric inpatient facility that has seen an increasing number of patients suffer from opioid addiction. A knowledge gap exists between current nursing approaches and adequate management of pain in opioid addicted patients. Nurses say the patients frequently do not have replacement therapy (methadone or suboxone) available upon their arrival at the hospital. Access to their doses is not easily obtainable depending on the time of day and the nurse's ability to contact the local methadone clinic. Attitudes can reveal nurse bias about a patient's appeal for pain medication. The bias judgment lies in a belief that the patient's intention is to request pain medications because of addiction rather than pain. 
Further evidence of nurses' challenges to effective pain management is revealed when patient satisfaction scores for the nursing unit are examined. Unit scores from a national survey company indicated dissatisfaction of in-patients with their pain management at the time of writing this proposal. It is important to consider the impact of dissatisfied patients with opioid addiction upon the survey results.

Psychiatric nurses who work at the $\mathrm{BH}$ unit are often faced with the angry and demanding behaviors of some opioid addicted patients requesting pain relief. Patient responses vary from perturbation to aggression. Patients are often not satisfied with available nursing interventions. The resulting poor outcomes are seen when patient satisfaction levels are surveyed.

Education on management of addiction for the unit nurses is identified as an opportunity. The opioid crisis has impacted the impatient setting as many more patients are dealing with heroin addiction than was seen previously. Nurses are vexed by completing narcotic withdrawal assessments that indicate the patient is suffering from withdrawal only because the patient "over-endorses." The nurse feels manipulated and the patient senses a negative attitude. Similarly, reporting of pain when the patient does not "appear" to be suffering from pain can affect the attitude of a nurse and subsequent experiences of the patient.

The target audience is identified as the 64 registered nurses employed on the 50-bed inpatient BH unit. This includes nurses who work forty hours per week, twenty-four hours per week and "as needed" nurses. They are associate degree and baccalaureate degree graduates.

The psychiatric nurses who work on the Behavioral Health Unit are diverse. They come from many personal and professional backgrounds and have unique experiences. In addition to the nurses with 5 to 15 years employed on the unit there are some nurses who were employed 25 or more years. Each nurse brings a unique flavor to the unit. The general feeling is one of kindness and caring when one examines their performance and display of integrity. Staffing turnover on this unit has traditionally been very low.

The nurses' compassion is not limited to their patients. They extend their civility to each other. This culture is supported by the Administrative Director's and the Unit Manager's consistent encouragement of staff to care for each other. Nurses on the BH unit are passionate about safe and evidenced-based care of the mentally ill. They are educated and trained in psychological and physical approaches to de-escalate patients who are upset and aggressive. The nurses comfort the delusional, hallucinating, suicidal and depressed individuals in their care while keeping them safe from harm. They encourage, educate and monitor. The nurses are flexible in their roles and work together to provide compassionate care for the extremely mentally ill.

\subsection{Problem Statement}

The psychiatric nurses lack adequate knowledge of opioid addiction as well as pain management strategies for patients. Attitude and bias interfere with delivery of adequate pain management and nursing care to those who suffer from opioid addiction.

\subsection{Description of Course}

The proposal is to teach the Behavioral Health nurses about opioid addiction and management of acute pain for those afflicted with opioid addiction. Many of these patients suffer with mental illness as well as opioid addiction, a condition known as "dual diagnosis." Education of the patient is also key to successful outcomes.

Psychiatric nurses easily engage in patient education because they are frequently talking with patients in place of performing physical care. Helping the patients takes the form of educating them about life management skills and mental health issues. The nurses need to understand biological and sociological considerations when educating someone with an opioid addiction.

The curriculum will also address opioid replacement therapy's impact on pain management so nurses can teach their patients what to expect. Addiction can be devastating. Individuals suffering from opioid addiction need information about the illness of addiction and measures to retrieve one's life from the edge of disaster. Patients with dual diagnosis are well represented on the Behavioral Health unit. Theirs is a complicated and multifaceted road to recovery. The class will address challenges to the nursing care of patients with dual diagnosis as it relates to unwanted behaviors among the opioid addicted.

Understanding the association of addiction and pain relief can assist in increasing nurses' empathy for patients with opioid addiction. Successful pain management has its foundation in the biology of pain. Nurses will be educated about how patients can respond to opioids in providing relief in the presence of acute pain. Finally, the presentation will address strategies to assist patients to accept education about how pain medication is dispensed 
at the hospital. Emphasis will be on approaches to minimize demanding and angry patient responses to their pain management approach.

The class will be held in two separate sessions lasting 45 minutes each. The first session will address addiction statistics, biological and sociological factors, and the humanitarian aspects of opioid addiction. The second session will focus on opioid replacement therapy and its relationship to pain management. The sessions will be offered eight times per week for two consecutive weeks. There will be eight sessions about addiction the first week and eight more sessions the next week for pain management. With this presentation schedule, all nurses can attend the two component sessions. The class sessions will be conducted at change-of-shift so the BH unit can be covered by both shifts. The classes will be in lecture form utilizing a power point presentation. The session will include experiential learning features throughout. For example, during the first session, discussion of patient behaviors will include a scenario followed by role play. The strategy of storytelling will be utilized in the second session when sending the message that opioid addiction can destroy individuals and their families. The learning strategy for each session will include question-and-answer periods in an informal setting for the nurses. The strategy of questioning the group and inviting verbal feedback and discussion will be utilized in the second session. A brief quiz to establish learning has taken place will be followed by a request for participants to complete an anonymous class evaluation after the last session.

As shown in Figure 1, the first step is to plan a meeting with the unit Administrative Director. Discussion will include the clear need for education as evidenced by psychiatric and hospital nurses voicing their concerns about opioid addiction and management of acute pain. Verbal permission to develop and present the class is solicited from the Administrative Director at this time.

The second step is to select a preceptor who has time, interest and energy to assist in this project. Someone with an educational background who is passionate about excellence in psychiatric nursing will be considered. Time constraints will be evaluated as well as degree level and experience. Permission of the organization granted by the unit administrative director and consent by the preceptor to participate will be obtained in writing.

The third step is to perform a literature search on the topic of opioid addiction and pain management to glean information that supports the educational plan implementation. This literature will be a foundation upon which to build the content of the curriculum. A literature search about teaching strategies will also be performed.

The fourth step is to develop the content of the curriculum to include information about addiction and pain management of those who are opioid addicted. The plan is to include education about the impact of dual diagnosis upon this patient population. The plan is also to develop teaching strategies for the first session that include lecture with power point presentation, storytelling, scenarios and role play. Likewise, the strategy of questioning the group and inviting verbal feedback and discussion will be employed throughout the second session.

The fifth step is to create a quiz to follow each session. Use of multiple choice and true/false questions will be employed for the advantage of familiarity to most participants. The plan also includes implementing a brief and anonymous evaluation form for each participant to complete following the quiz. Preparation for this step includes sending both a copy of the quiz and of the evaluation to the hospital print shop for multiple copies.

The sixth step is to address support services needed to present the sessions. This includes reservation of the conference room on the BH unit in which to present the sessions. A back-up classroom will be reserved off the unit in case the conference room is suddenly occupied that session. Support service also includes procurement of a projector and computer set-up from the audio-visual department for the two-week period the sessions will be presented.

The seventh step will be actually developing the two power point presentations for the psychiatric nurses using the content gleaned from research of the literature. The power point will be augmented with graphics, animation, and art to keep the presentation fresh and interesting. A meeting will be scheduled with the IT educator at the hospital to gain access to printable products such as clip art and photos needed to create the slides.

The final step is to create flyers and prepare emails to make available to all registered nurses employed on the $\mathrm{BH}$ unit. The times and dates of the sessions will be documented on the daily assignment sheets the week prior to the onset of sessions. Organization-required sign-in sheets will be prepared for the sessions for the purpose of tracking attendance. 


\subsubsection{Planning and Implementation Timeline}

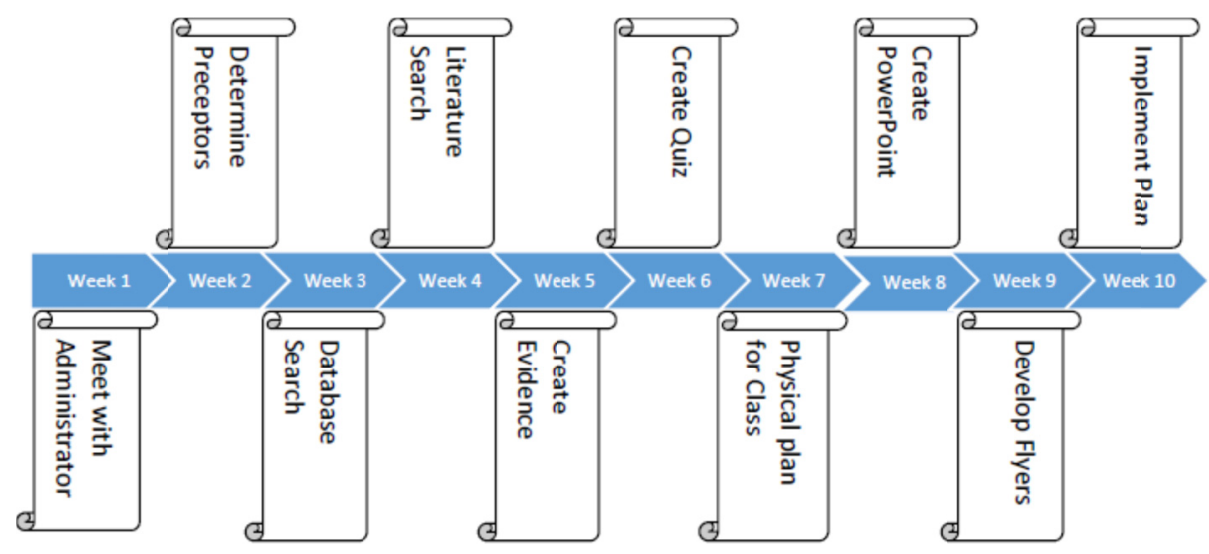

Figure 1. Timeline of planning and implementation

The first week will be devoted to scheduling a meeting with the Administrative Director of the BH unit. By the end of this week the Administrative Director should be briefed on the idea of presenting an educational program to the psychiatric nurses about management of pain in opioid addicted patients. Meeting content should include obtaining agreement to sign the Organizational Approval Letter.

The second week will be used to select a preceptor. Prerequisites include time available, interest in the topic, and passion for excellent psychiatric nursing. Opinions of the administrative director and unit manager should be sought regarding possible candidates. Once selected, the preceptor should sign the Preceptor Agreement. By the end of this second week, the Organizational Approval Letter should also be signed by the administrative director of the BH unit.

The third and fourth weeks will be devoted to a literature search that supports the educational plan to cover the gap in education for nurses related to pain management of opioid addicted patients. At least five of the articles selected should be scholarly and peer reviewed. The articles chosen should be limited to those published within the past five years. Cutting-edge resources should be sought to bring new ideas and material to the educational experience for the nurses. The literature search results should be reviewed with the preceptor to gain perspective and advice about the articles.

The fifth week will be filled with creating the evidence summary of the literature. Topics covered should include a description of opioid addiction and its impact upon acute pain control. The impact of dual diagnosis should be included. The summary should be informative and relative to the topic. Completion of this summary should provide an interesting content for the class. The evidence summary should be provided to the preceptor for review.

The sixth week will address creation of a quiz using multiple choice and true/false questions. An evaluation of the class should be developed. The quiz and evaluation should be one page each. The quiz should be reviewed with the preceptor for critique.

The seventh week is devoted to physical planning for the class events. The room should be reserved at this time by signing up on the designated monthly calendar. A call should be made to audio visual communications to reserve a projector and computer for the proposed power point presentations lasting for a two-week period. A back-up classroom should be reserved in case an emergent need for the BH conference room should occur.

The eighth and ninth weeks will be spent creating the two separate power point presentations for the first and second class. The power point slides should be simple, brief and colorful. Print font should be of a legible size from a distance. Graphics and photos should be visually appealing. A consultation with the hospital Internet Technology (IT) nurse educator should be made to access photos, animations or graphics to insert into the slides. Copies of the power point presentations should be provided to the preceptor for approval.

The tenth week brings the preparation to a close as flyers are developed then posted and emails are sent to announce the upcoming classes. Reminders of the classes and times available should be written in red ink on the daily assignment sheets for nurses. Attendance sign-in sheets should be printed and available for the classes. 


\subsection{Rationale}

During a study of patients, those with opioid addiction felt they were being "treated like a junkie." They reported feelings of not being respected by the approach of nurses. The patients said they felt they had experienced contempt from the nurses (Morley, Briggs, \& Chumbley, 2015, p. 703). Such maltreatment comes from bias of nurses. It is not a representation of a good patient experience in modern healthcare. The rationale for educating psychiatric nurses about opioid addiction and management of the acute pain of those with addiction is reflected in the attitude change that increased knowledge will bring.

Patients with Substance Use Disorder (SUD as in opioid addiction) are at greater risk of suffering severe pain because of comorbidity with other disorders. These include opioid-induced hyperalgesia and withdrawal from the opioids. Additionally, the patient in pain with SUD may demonstrate difficult-to-manage behaviors that can result in stigma and labelling by nurses (Morley et al., 2015). Nurses armed with new knowledge about an age-old condition can make a difference in the way opioid addicted patients are treated.

Successful outcomes to the education of the psychiatric nurses on the BH unit will be demonstrated by the knowledge gained during the class. Students of the class will learn about opioid addiction and how nurses can manage acute pain when the patient is opioid addicted.

The result of this new knowledge is increased confidence as the psychiatric nurses on the BH unit develop new skills and techniques. As the nurses become more comfortable and successful with the care of this patient population, the patients will perceive assistance rather than resistance to managing their pain. Patients with opioid addiction will receive kind, compassionate and intelligent management of their acute or chronic pain. This will be accomplished with education of the patient and increased understanding on the part of the nurse. Additionally, the anticipated result of the education is for improved patient satisfaction scores for management of pain that will justify the rationale.

\section{Review of the Literature}

Ten credible sources were reviewed to develop the foundations for presenting a class for psychiatric nurses about pain management in opioid addicted patients. The criteria for choosing these articles was that they were peer reviewed, published from 2011 to 2018, and scholarly.

The psychiatric nurses on the BH unit need to know the fundamentals of opioid addiction. This will engage the nurses in the idea that addiction is an affliction with a biological foundation. The nurses need to know how to achieve adequate pain management in individuals who suffer with opioid addiction. Management of pain will include patient education and psychological support. Additionally, the nurses need to know about dual diagnosis and its impact on patient behaviors.

Countless individuals maintain the notion that addiction is the result of a person's moral turpitude. The bias clearly exists, even among medical professionals. Literature reviewed consistently refers to scientific evidence indicating addiction is a disease (Lembke, 2018). The development of opioid addiction as a disease is hypothesized in the Brain Disease Model of Addiction (BDMA.) This model describes change following drug use in the chemistry of the brain that is only reversed with difficulty (Meurk, Fraser, Lucke, Carter, \& Hall, 2016). The disease model of addiction removes stigma, makes addiction a legitimate illness and demands health insurance and research funding be made available (Lembke, 2018).

Those who study the brain when addiction takes place find there is a neurochemical change in the brain. Opioid chemicals interrelate with receptors in the nerve cells of the brain and body. Because euphoria is one of the main effects, other than relief of pain, the patient craves higher doses and may take more than the physician has prescribed. This can lead to dependence upon the drug and addiction when misused. The indiscriminate use of opioids can lead to overdoses and death (National Institute on Drug Abuse [NIDA], n.d.). Meanwhile, the addicted individual requires more and more of the opioid to meet their physiological and psychological needs.

The individual with addiction suffers from two main symptoms. The first is tolerance. The victim will need higher and higher doses of the drug to prevent the agony of the second symptom: withdrawal (McCoy, 2014). Symptoms of withdrawal include diaphoresis, pain in the abdomen, nausea, and bouts of diarrhea (Shi, Kandola, Tobey, \& Singer, 2017). As the withdrawal progresses the patient experiences muscle and bone pain with restlessness. In addition to nausea there is vomiting and cold flashes with pili-motor reflex demonstrated (also known as "goose bumps.") There is leg jerking beyond voluntary control and the individual experiences insomnia (National Institutes of Health [NIH], 2018).

Sociological implications must be examined by psychiatric nurses as the families and loved ones of addicts suffer terribly when the person is in the throes of addiction. The addict loses economic standing and often 
alienates friends and family as they spiral downward in attempts to stay ahead of the drug cravings. Teaching family members about the science of addictions has shown some promise in easing the angst about their loved one's condition (Meurk et al., 2016). It is anticipated that the psychiatric nurses on the BH unit will experience the same understanding.

Currently addiction is at an all-time high. People are dying from opioid overdoses at an alarming rate (Lembke, 2018). In May of 2017 the state of Ohio filed a lawsuit against several pharmaceutical companies for their part in the opioid epidemic that has drained the Medicaid funds in that state (McCoy, 2014). Political representatives who may lack adequate knowledge of pain control and opioid dependence issues are changing laws. These laws and subsequent corporate changes surrounding availability of opioid products affect millions of those who consistently take their medications as directed by their physician. These patients are being left with few choices for chronic pain control. Successful pain control for them may have been in place for years with extended relief opioids. Now legitimate access to these medications has decreased as pharmaceutical companies cease production and insurance companies refuse to pay (J. Roth, personal communication, March 20, 2018).

The United States consumes $80 \%$ of the world's opioids yet represents only $5 \%$ of the population of the planet. There are about 40 million people who suffer from addiction in the United States representing $16 \%$ of the population. The number of Americans who are affected is more than those with diabetes ( 26 million,) with heart disease (27 million,) and with cancer (19 million.) The number of individuals dying from addiction is growing (Lembke, 2018). The scope of heroin use alone is staggering. The National Survey of Drug Use and Health (NSDUH) reported in 2016 that 948,000 people in the United States admitted to having used heroin during the previous year. The number of people trying heroin is appalling as another 170,000 tried heroin for the first time in 2016. Prescription opioid abuse compared to an increase in heroin use in the U.S. is being examined (National Institute on Drug Abuse [NIDA], 2018).

Bias on the part of the nurse can be the first hurdle to overcome when achieving adequate pain management for patients suffering from opioid addiction as well as acute pain. Review of the literature indicates that attitudes on the part of nurses can be reflected in the timing of the pre-intervention assessment compared to when the patient requested pain relief medication. Bias was found when nurses avoided making prompt responses to requests for pain medication when the patients appeared to be free from pain causing events or disease processes (Schreiber et al., 2014). It is assumed these nurses avoid administering the medications for longer time periods when the patients were deemed drug seekers, suicidal, readmitted at frequent intervals and elderly with confusion. Under these conditions, limited insight was utilized for patients who were perceived to be experiencing pain judged "without a physical cause" (Schreiber et al., 2014).

A phenomenological study of hospital nurses was performed in which the researchers questioned nurses about their practice when caring for patients with addiction history in pain. Deemed "drug seekers," these patients were described as being "difficult" by hospital nurses. Nurses interviewed were encouraged to share their thoughts about the patient with opioid addiction when these individuals complain of pain. The nurses reported that the patients with addiction presented challenges to providing care. One nurse shared that the patients with addiction were demanding. They asked for their pain medication at the exact times due, exhibited manipulative behaviors and became verbally aggressive with the nurse. Other nurses added that these patients tend to be non-compliant in their care regimen (Morley et al., 2015).

In an earlier study, patients felt they were "treated like a junkie." They reported feelings of not being respected or experiencing contempt from the nurse (Morley et al., 2015, p. 703). Patients with opioid addiction are at greater risk of suffering severe pain because of co-morbidity with other disorders. These include opioid-induced hyperalgesia and withdrawal from the opioids. Additionally, the patient in pain with addiction may demonstrate difficult-to-manage behaviors that can result in stigma and labelling by nurses (Morley et al., 2015).

When opioid addicted patients suffer acute pain, replacement therapy must be in place before opioid pain medication can be added and expect to bring relief (Sen et al., 2016). Establishing a safe dose that will relieve pain includes obtaining a current dose of opioid replacement medication from the patient's health provider or clinic. This is the number one priority in pain management for the nurse as soon as the patient is admitted. The receptor sites are many because of the high tolerance of opioids seen with continued misuse (Sen et al., 2016). The patient can receive relief from acute pain post-operatively, with deep lacerations, burns and other self-inflicted injuries as seen on the BH unit. However, the process is arduous, especially when influenced by the current panic about the opioid epidemic. It is physician driven and is dependent upon a complete and unbiased history from the patient that involves gaining trust. The patient must be receiving replacement therapy before opioids are ordered for the management of acute pain or the opioids simply will not work (Sen et al., 2016). The 
psychiatric nurse must also gain trust to obtain an accurate history and teach the patient about limitations related to nurses' response to doctor's orders. Psychiatric nurses will learn to educate the patient about the disease model of addiction. The nurse should present a compassionate approach to care. Furthermore, the nurse would be wise to be prompt when administering pain medications to patients whose receptor sites are sensitive to omission (Sen et al., 2016). Psychiatric nurses on the BH unit have access to a locked "sensory cabinet" that offers distracting activities, music delivery and soothing oils as needed to augment pain management of all patients.

Finally, the psychiatric nurses are obligated to assess the impact of opioid abuse on those with serious mental illness. These individuals suffer from what is known as "dual diagnosis." Care of those with dual diagnosis is challenging. Literature suggests that these patients frequently have worse prognoses than those without a co-morbid disorder (Prodromou, Kyritsi, \& Koukia, 2014). Nursing intervention includes active listening to the patient's reported needs. It is important to avoid bias on the part of the nurse by insinuating the nurse knows what is best. The nurse should utilize unique and creative ways to deliver person-centered care and avoid neglecting individuals based upon their co-morbid illness (Fieldhouse \& Wilson, 2017).

\subsection{Best Practices}

The psychiatric nurses on the $\mathrm{BH}$ unit want evidence based material to guide their practice. They want assurance the material they teach their patients is truthful and proven. The content for the classes is drawn from information from peer-reviewed and scholarly articles. These articles promote ideas about bias and its impact upon nurses and their care (Schreiber et al., 2014). The best way to combat stigma and bias is with education that includes scientific evidence that opioid addiction is a disease (Lembke, 2018). Symptoms of this disease include tolerance of the drug and withdrawal from the drug (McCoy, 2014). Statistics show the negative impact of opioid addiction upon individuals, families and communities (Meurk et al., 2016). The lure of the euphoric effects of opioid abuse is too much for many to manage without responding to biologically induced cravings to take in more (National Institutes of Drug Abuse, [NIDA], 2018).

Nurses need to know that acute pain in individuals suffering from opioid addiction is medically treatable (Sen et al., 2016). Much of their frustration is linked to helpless feelings about an inability to provide adequate pain relief to their patients with opioid addiction. Most nurses crave the opportunity to interact with others on the healthcare team about improving patient care delivery. The idea of opioid replacement therapy based on evidence found in the literature is an excellent foundation to start a conversation about acute pain management (Sen et al., 2016).

\subsection{Recommendation}

The problem of a knowledge gap about acute pain management of patients with opioid addiction is acknowledged by the psychiatric nurses on the $\mathrm{BH}$ unit. Pain management scenarios the nurses face on the $\mathrm{BH}$ unit include post-care of severe injuries sustained during an attempt at suicide. Injuries the nurses mention responsible for inducing acute pain are deep lacerations, severe burns, and fracture injuries. Inadequate educational support has been provided to the nurses on the topic of pain management in the opioid addicted patient population. Bias among the nurses is possible.

To rectify the situation, it is recommended the BH Nurse Educator develop classes that educate the psychiatric nurses about the stigma faced by patients with opioid addiction. Additionally it is recommended the nurses learn how to participate with the interprofessional team to better manage acute pain.

The class should illustrate the need for both the patient and the nurse to know that addiction is an illness based on a disease model (Lembke, 2018). It is further recommended that the nurses be educated about the neurochemical disease process of opioid addiction. The most recent evidence based on medical treatment for those with opioid addiction should be discussed (Sen et al., 2016). This includes treatment with replacement therapy so that opioids can be successfully ordered and actually provide relief. The psychiatric nurses need to embrace patient education as a means to combat the myths and biases against addiction.

Finally, the vital information about pain management in opioid addicted patients communicated to the psychiatric nurses on the BH unit is to be evidenced based. Literature has been searched and reviewed to identify the impact of disease knowledge upon opioid addiction as a pathway to improved patient care and patient education. Having one's pain management implemented appropriately leads to a better patient experience and providing adequate pain management is a better experience for the nurse.

\section{Project Launch: Steps}

The first step was to meet with the Behavioral Health (BH) unit Administrative Director. A discussion did occur, and the Administrative director was informed of the concerns expressed by the unit and hospital nurses about 
opioid addiction and management of acute pain. Verbal permission to develop and present a class for the behavioral health nurses was solicited. The Administrative Director was supportive of the project idea and enthusiastic about the potential for positive outcomes for the unit's patients and nurses and then agreed to sign necessary documents.

The second step was to select a preceptor who had the time, interest and energy to assist in this project. A nurse was selected who has experience and background in psychiatric nursing education. She is passionate about excellence in psychiatric nursing. The preceptor candidate has a master's degree in nursing and she is a seasoned nurse of several decades. She is currently employed as a psychiatric Nursing Clinical Instructor at two local institutions of higher learning: a university and a community college. She embraced the request to become a preceptor with grace and intense interest. She was excited about selection of the topic of pain management for opioid addicted patients.

The third step was to perform a literature search on the topic of opioid addiction and pain management with an emphasis on nursing care. The purpose was to collect information that supports the education plan implementation. Many articles were reviewed to develop a foundation upon which to build the content of the curriculum. The search was limited in time and scope specific to the rubric. A literature search about teaching strategies was also performed to provide insight and inspiration for the teaching style of the classes.

The fourth step was to develop the content of the curriculum to include information about the condition of addiction and pain management of those who are opioid addicted. The content includes material about the impact of dual diagnosis upon this patient population. The content also includes information about stigma and bias in the addicted patient population. Teaching strategies have been reexamined during this step as well. The plan remains to use power point presentation during the first-class lecture that involves presenting a scenario to which staff will role play. The plan for the second class remains to utilize questioning the group and inviting verbal feedback and discussion. The discussion will center on an addicted patient's story.

The fifth step is to create a quiz to assess learning of the nurses attending the classes. The plan is to use a combination of multiple-choice questions and true/false questions. These will be employed for the advantage of familiarity to most participants. The plan is to also create a brief and anonymous evaluation of the classes after the quiz. This data will be used to improve subsequent presentation of the classes.

The sixth step is to address support services needed to present the classes. This includes reservation of the conference room on the $\mathrm{BH}$ unit in which to present the classes. A back-up classroom at the Education Center will be reserved off the unit in case the conference room is suddenly occupied at class time. Support service also includes procurement of a projector and computer set-up from the Education Center for the two-week period the classes will be conducted.

The seventh step will be the actual development of the two power point presentations for the psychiatric nurses using the content gleaned from research of the literature. The power points will be augmented with graphics, animation and art to keep the presentations fresh and interesting. A meeting will be scheduled with the Clinical Technology Nursing Educator at the hospital to gain access to printable products such as clip art and photos needed to create the slides.

The final step is to select actual dates, create flyers and prepare emails to all registered nurses employed on the $\mathrm{BH}$ unit. The times and dates of the classes will be documented on the daily assignment sheets the week prior to the onset of classes. Organization-required sign-in sheets will be prepared for the classes for the purpose of tracking attendance.

\section{Identification of Inter-professional Relationships}

The Administrative Director of the BH unit has great influence over the project. The BH unit's Medical Director has the greatest impact on the group of residents and attending psychiatrists. BH staff nurses are key stakeholders in the project. Included as an important interprofessional collaborator is their Nurse Manager, a dedicated servant leader. Audio/visual technical support is provided by the Director of the Education Center. The Clinical Technology Nurse Educator is called to join the team to assist with enhancement of the power point presentations. Fellow educators, especially the hospital librarian, are enthusiastic supporters of the project. The preceptor is an outstanding choice.

\section{Discussion of Relationships}

The behavioral health $(\mathrm{BH})$ unit Administrative Director fully endorses completion of this project. She sets high standards for her nurses to achieve excellence in care. She is a superb nursing leader. Essential to the project has been her support and ambition for the classes to be conducted in the most reasonable time-frame and with the 
most accurate and current information. She heartily supports the endeavor for the sake of her patients and nurses. She can gather resources and gain access to services required to conduct the classes. She makes financial decisions about staffing costs to cover the nurses' overtime pay as is required in order to present the classes.

The administrative Director works closely with the unit psychiatrists to coordinate all patient care services for the 50 bed $\mathrm{BH}$ unit and fifteen psychiatric screeners in the Emergency Department. The Medical Director especially collaborates with the Administrative Director as their goal continues to be delivery of excellent care by all $\mathrm{BH}$ care providers. The same hurdles faced by nurses are also faced by the physicians concerning stigma and bias when caring for those with opioid addiction.

Staff nurses can determine the project's success by their attitudes as they complete the classes. The psychiatric nurses on the BH unit are a close-knit group. They will share their opinions about the classes with co-workers who have not yet attended the class. Positive feedback to peers will help to promote successful implementation. Negative feedback could present the educator with challenges as the classes proceed. The BH unit Nurse Manager can be counted on to support the project to her fullest. Her positive attitude with staff will likely prevail over any negative attitudes that may develop.

The Director of the Education Center provides audio-visual services to the hospital. He assures a projector and computer cart will be available for the two-week period of presentations. Contact has been made with the Education Center's classroom scheduler for back-up availability should the BH unit conference room be commandeered on the days of the classes. Fellow Nurse Educators have been instrumental in providing preparation and presentation tips for implementation of the classes.

The preceptor has been extremely helpful in developing the lesson plan and very supportive of the project's focus. She recommended the hospital librarian to assist with perfecting APA references. Corrections were completed successfully.

\section{Organizational Sustainability and Implementation Support}

The short-term goal is to successfully complete the two weeks of classes no later than September of this year. The Administrative Director will approve the content of the presentation. She gives permission to schedule and present the classes to the psychiatric nurses on the BH unit. She authorizes overtime pay for nurses for attending the classes. She deems the classes be mandatory. She states the classes must be provided for new BH nurses during unit orientation competency sessions after September. This directive enables all nurses employed on the $\mathrm{BH}$ unit to be educated about pain management of opioid addicted patients. Both seasoned and new nurses will be provided an opportunity to examine possible bias on their part and learn about the disease model of addiction.

In addition to education on the $\mathrm{BH}$ unit, the long-term goal has always been to present an edited version of the classes to include all nurses at the hospital. The annual competency fair is provided six times per year by the hospital. It has been traditional these past few years to present new topics at the fair each year. It is hoped and anticipated that this topic will be chosen for next year's fair. Competency in pain management of opioid addicted patients can be guaranteed no further than that because the fair presentations are currently not included in new nurse hospital orientation. This is related to the hospital requiring a recently introduced and very specific onboarding education with lesson plans that are standardized nation-wide. The plan from there is to propose an additional day of orientation/ onboarding to that provided by the mother company. Members of the hospital educator team can be counted on to support such an endeavor. The educators recognize the need for other local hospital initiatives to be shared with incoming nurses, such as an introduction to library services and video monitoring systems to prevent falls.

\section{Resources for Implementation}

The project requires minimal direct financial support due to availability of hospital resources such as a classroom, audio-visual equipment and the print shop which are all received at no extra cost to the BH unit. Nurse coverage of the $\mathrm{BH}$ unit will be accomplished with afternoon, evening and day shift nurses relieving the participants from duty for the twice daily classes.

The rearrangement of staff during the shift change sessions is likely to be a budget burden because of the additional 40 minutes past end-of-shift time accrued for each participant. The greatest expense is the overtime pay afforded the nurses as is required for mandatory education. This is a particularly sensitive issue at this time due to corporate financial cutbacks related to political impacts on healthcare economics. The Administrative Director of the $\mathrm{BH}$ unit is committed to this important and vital information as it relates to the current opioid epidemic and will approve the overtime pay. 
Precious time from busy health care staff is required to successfully complete the project. The Administrative Director is responsible for five different departments as well as having hospital administrative duties. Her generous time spent discussing the project and agreeing to its implementation is commendable.

The Director of the Education Center provides the projector and computer cart and will allow use of the equipment for the two weeks required. The Clinical Technology nurse educator is called upon to assist with clip-art and photographic additions to the power point slides. Her skill set is selected because her quality contributions to nursing education have been typically interesting and stimulating.

The Preceptor is highly engaged in the project and volunteers her time to meet with the BH nurse educator. She reviews progress at regular weekly intervals. Her efforts are immeasurable as she makes personal adjustments in her busy schedule as a psychiatric nursing clinical instructor to meet.

Equipment used for the project includes the BH educator's personal computer and printer. Web access is available using this personal computer for a university library literature search. The copy machine on the BH unit is needed for scanning and sending important documents related to the project. The educator's work phone and personal cell phone are needed for verbal communications with the preceptor between meetings. A projector cart with computer is required for presentations conducted on the $\mathrm{BH}$ unit. The conference room already has a viewing screen on the wall. Finally, a flash drive is required for power point presentation portability.

Classroom space is available in the conference room on the BH unit. This is the only conference space on the unit that is not a patient care area. Should an emergency develop that requires use of the room by the Administrative Director, an alternate classroom space is prudent. This will be accomplished by reserving a classroom in the Education Center for each session if the need arises. It requires a three-minute walk to this alternative and each room is equipped with a projector, computer and screen.

\section{Discussion of Outcomes}

The intended outcomes of the project will be first evaluated when the results of the quizzes indicate by data that learning about the disease model of addiction has taken place. The quiz may also reveal gaps in learning not previously identified. Another outcome shown will be Behavioral Health $(\mathrm{BH})$ nurses who have taken the classes being more sensitive to the feelings of those with addiction. For example, the practice of referring to opioid addicts as "drug seekers" will disappear from the vocabulary of psychiatric nurses on the BH unit. Acute pain in opioid addicts will be managed by an interprofessional team of advocates for a solution to the patient's suffering. This will happen when nurses are seen to model communication with physicians and therapists that supports addiction as a disease. A dialogue about opioid replacement therapy to manage acute pain should result.

Additional outcomes are identified as performance of and documentation of education provided to the patient about the disease model of addiction by the nurses. Continued education should reflect patient learning about bias and stigma. An outcome would be the patient would be willing to try opioid replacement therapy to help manage his or her pain. By giving positive reviews of nursing care provided during their hospital stay on the $\mathrm{BH}$ unit, patients could indicate an improvement in how those with opioid addiction are treated.

After completing the classes, the nurses will be observed to impact care delivery when having a conversation with the patient. They will be encouraging participation in medical treatment of addiction. The patient must be willing to participate in opioid replacement therapy before pain management can be effectively delivered by the doctors and the nurses. Psychiatric nurses on the BH unit will be seen to provide comprehensive and patient-centered discharge information about addiction, bias, stigma, and acute pain management when the patient is leaving the hospital. Another outcome observed at discharge would be reassurance by the nurse that recovery from addiction is possible.

\section{Impacts on Future Outcomes}

The future of pain management of opioid addicted patients with acute pain is bright. Nurses and physicians will seek to work together to gather pertinent intake information at admission and disseminate meaningful information about addiction and its treatment by the time of discharge. Attitudes about the status of those suffering from opioid addiction should improve. Patient centered care will include evidence of elimination of bias and nurses taking a stand against stigma.

Opioid addicted patients should obtain effective pain management when they are suffering from acute pain such as from injuries sustained during a suicide attempt. This achievement would be a positive outcome for patients, nurses and physicians in the future. Patients provided adequate pain management on the BH unit who suffer from opioid addiction may indicate their gratitude through improved patient satisfaction surveys. Satisfaction with pain management has typically remained below unit goals. 
Finally, the future holds the opportunity for patient education delivered by nurses to impact the vulnerable adults suffering from addiction everywhere we serve. A grass roots movement by nurses empowered with knowledge and sensitivity can change destructive attitudes about those suffering from opioid addiction in health care settings, communities, states and the nation.

\section{Identification of Inter-professional Participants}

Feedback was received by the BH Nurse Educator from nursing staff that the patients with opioid addiction presented a dilemma for them in terms of providing adequate pain management and dealing with difficult behaviors. This information was brought to the attention of the Administrative Director of the BH unit in a meeting wherein the BH Nursing Educator revealed a plan to develop an education piece around management of pain in opioid addicted patients. The project idea was approved by the Administrative Director. The Administrative Director states she based her decision on literature with which she was familiar describing the opioid epidemic. She assessed this information as it relates to opioid addicted inpatients on the BH unit. In the planning stage of her decision she deemed the classes should be comprehensive, mandatory and pertinent to the plight of the opioid addicted individual. She expressed trust in the BH Nursing Educator's ability to produce an interesting educational piece for the $\mathrm{BH}$ unit nurses concerning this topic. This was based on previous examples of the Nurse Educator's work.

The Administrative Director's decision-making process also included helping the BH Nurse Educator select a preceptor for the project. Both she and the $\mathrm{BH}$ unit Nurse Manager were ruled out as preceptors due to time constraints both personal and professional on each. The Administrative Director thoughtfully recommended the psychiatric Clinical Nursing Instructor as a candidate who is known as a great collaborator among the BH staff for many years.

The preceptor assisted the BH Nursing Educator to narrow the topic and to examine the steps involved in the proposal to be developed. For example, an idea of the BH Nurse Educator to include an extensive section devoted to dual diagnosis was reconsidered due to the preceptor pointing out the volume of information already planned. This decision was related to an abundance of addiction and pain management information identified during the literature review.

Decisions made in consultation with the preceptor also included providing two separate 45 minute sessions to cover the information needed to assist nurses in their goal of patient centered care to opioid addicted patients.

Finally, the preceptor's suggestion that the hospital librarian be contacted with various formatting issues was based on her long-time familiarity with hospital resources unknown to the BH Nursing Educator. The skillset of the librarian resulted in satisfactory formatting of the project. Such collaboration maintained a cohesive set of players involved in the completion of the project to educate psychiatric nurses about acute pain management of opioid addicted patients.

\section{Inter-professional Collaboration}

Before the presentation is approved, the Administrative Director and the Medical Director must meet and decide if the project is appropriate. The BH Nurse Manager will support her staff attending the classes and monitor verbal feedback for the nurse educator. She supports the mandatory status of the education and its inclusion in new nurse orientation for the BH unit. She partners with the Nurse Educator to generate enthusiasm for the classes with her staff. The staff will faithfully attend mandatory nursing education and participate fully as is their pattern. The power point slides will be developed with the assistance of the Clinical Technology Nurse Educator. Practice presentation of the two classes will be attended by the unit Nurse Manager as arranged.

Utilizing the influence of the Administrative Director of the $\mathrm{BH}$ unit and the experienced assistance of the Clinical Technology Nurse Educator, a new project will be approved and developed. This will center on the creation of an on-line learning lesson about pain management of opioid addicted patients in the hospital setting. The Clinical Technology Nurse Educator will collaborate with her peers to convert the project into an on-line learning lesson for all nurses in the company. It will address the concepts outlined in the project.

\section{Closing the Gap}

Education of psychiatric nurses on the BH unit will be the pathway to educating patients with opioid addiction about the disease process of addiction. Bias and stigma need to be addressed and conquered (Morley et al., 2015). Nurses with knowledge of current treatment trends in the management of acute pain can model communication with physicians about opioid replacement therapy. The idea that addiction is a disease with treatment available and recovery possible is essential for opioid addicts, their families, and their nurses to comprehend (Lembke, 2018). Through rehabilitation and medical intervention with opioid replacement therapy patients can be helped 
to recover their lost lives. Education of the patient will help to eradicate bias and stigma as attitudes melt about individuals with opioid addiction.

The literature shows there remains bias among nurses who do not understand that addiction is a disease (Morley et al., 2015). There are nurses who secretly believe pain can be assessed accurately by the nurse. The nurse misses an opportunity to improve the patient experience. The response directly conflicts with the patient's presentation and verbal complaint of pain (Schreiber et al., 2014).

The psychiatric nurses on the BH unit will be educated about how bias and stigma impact negatively upon the lives of opioid addicts (Morley et al., 2015). They will learn how it can interfere with patients receiving adequate pain management for acute injuries. Finally, the classes will emphasize that management of acute pain in opioid addicts is treatable only with opioid replacement therapy first (Sen et al., 2016).

\section{Reflection}

The expected outcome of this project was: "Assemble scientific findings from nursing biopsychosocial fields, genetics, public health and organization sciences for the continual improvement of nursing care across diverse settings."

Central to the project, the literature review provided a rich source of information about opioid addiction and management of acute pain in patients who suffer from opioid addiction. Scientific findings from the literature review led me to investigate biopsychosocial fields of study about addiction and to learn about the public health attention to the current opioid epidemic. The gap in education for nurses on the Behavioral Health (BH) unit revealed a lack of education about pain management, especially for those patients with opioid addiction.

The focus of the project is to teach nurses how to educate patients, overcome bias and stigma and to manage acute pain for patients with opioid addiction. The plan includes education to current nurses and continuing education for those hired in the future. Future plans include education of nursing staff about opioid addiction and pain management being extended throughout the hospital. Patients who suffer from opioid addiction originate from diverse backgrounds and settings. Their lives are further complicated when the patient suffers a major mental illness. The stigma of addiction is pervasive. It is the conviction of the BH Nurse Educator that management of their pain must be supported by our faith-based hospital ministry whose mission statement calls for compassionate care to the poor and vulnerable. "Serving the least of these" exemplifies a culture of excellence.

The second outcome integrated into the project was: "Construct interprofessional teams to communicate, coordinate, collaborate and consult with other health professionals to advance a culture of excellence."

Integration of this outcome is revealed when the third competency of the Lesson Plan is reviewed. This competency calls for the psychiatric nurse to "model communication with members of the health care team to be an advocate for management of acute pain in opioid addicted patients." Accessing newly acquired knowledge about opioid addiction and opioid replacement therapy, the psychiatric nurse can assist her patient to a path of education about addiction, bias and stigma. The nurse can address acute pain management with the psychiatric physicians and advocate for a thorough assessment of the pain management needs of the opioid addicted patient.

Seeking a solution to a terrible problem of failure to adequately manage the acute pain of patients with opioid addiction on the $\mathrm{BH}$ unit, I have reached the conclusion that opioid replacement therapy should be considered for all those suffering with addiction. Opioid replacement therapy seems the only answer for individuals in need of rehabilitation to end their burden of addiction. It should be accessible to any person with addiction to opioids in every healthcare setting.

\section{Acknowledgement}

Dedicated to Deborah Lynn Russell without whose help this paper would never have been completed. Sincere thanks to Helen Palleschi, who served as an outstanding preceptor and gave me encouragement and motivation in this journey.

\section{References}

Fieldhouse, C., \& Wilson, S. (2017). EIDDER: Early intervention dual diagnosis engagement and recovery. Foundation of Nursing Studies, 13(1).

Lembke, A. (2018). Why addiction should be considered a disease. Judges' Journal, 57(1), 4-8.

McCoy, K. M. (2014). Opioid abuse. Encyclopedia of Health. Salem press. 
Meurk, C., Fraser, D., Lucke, M., Carter, A., \& Hall, W. (2016). Neurological explanations in accounts of a family member's addiction. Drug and Alcohol Review, 35(4), 461-469. https://doi.org/10.1111/dar.12318

Morley, G., Briggs, E., \& Chumbley, G. (2015). Nurses' experiences of patients with substance-use disorder in pain: A phenomenological study. Pain Management Nursing, 16(5), 701-711. https://doi.org/10.1016/j.pmn.2015.03.005

National Institute on Drug Abuse. (2018). Heroin: What is the scope of heroin use in the United States? Retrieved from https://www.drugabuse.gov/publications/research-reports/heroin

National Institute on Drug Abuse. (2018). Opioids. Retrieved from https://www.drugabuse.gov/drugs-abuse/opioids

National Institutes of Health. (2011). Opioids and chronic pain. Retrieved from https://medlineplus.gov/magazine/issues/spring11/articles/spring11pg9.html

Prodromou, M., Kyritsi, E., \& Koukia, E. (2014). Dual diagnosis affects prognosis in patients with drug dependence in integrative care setting. Health Science Journal, 8(2), 216-228.

Schreiber, J. A., Cantrell, D., Moe, K. A., Hench, J., McKinney, E., Lewis, C. P., ... Brockopp, D. (2014). Improving knowledge, assessment, and attitudes related to pain management: Evaluation of an intervention. Pain Management Nursing, 15(2), 474-481. https://doi.org/10.1016/j.pmn.2012.12.006

Sen, S., Arulkumar, S., Cornett, E. M., Gayle, J. A., Flower, R. R., Fox, C. J., \& Kaye, A. D. (2016). New pain management options for the surgical patient on methadone and buprenorphine. Current Pain and Headache Reports, 20(3), 16. https://doi.org/10.1007/s11916-016-0549-9

Shi, C. R., Kandola, M. S., Tobey, M., \& Singer, E. (2017). Managing opioid withdrawal for hospital patients in custody. Hastings Center Report, 9-10. https://doi.org/10.1002/hast.683

\section{Copyrights}

Copyright for this article is retained by the author(s), with first publication rights granted to the journal.

This is an open-access article distributed under the terms and conditions of the Creative Commons Attribution license (http://creativecommons.org/licenses/by/4.0/). 